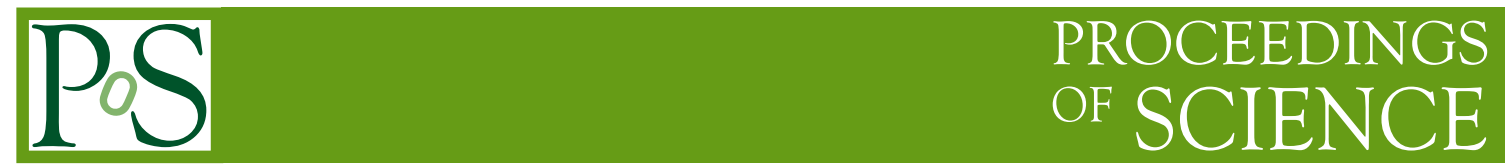

\title{
Improving hadron creation operators on the lattice
}

\section{Mike Peardon*}

School of Mathematics,

Trinity College Dublin,

Ireland

e-mail: mjpemaths.tcd.ie

\begin{abstract}
A new gauge-covariant smearing algorithm for quark fields is described. The method applies a low-rank operator to define the smooth fields to be used in constructing hadronic creation operators. Since the space of smooth fields is small, all elements of the reduced quark propagator can either be computed exactly or estimated efficiently. The algorithm enables arbitrary sources to be inserted in correlation functions, including multi-hadron operators. First results from the method are presented.
\end{abstract}

The XXVIII International Symposium on Lattice Field Theory, Lattice2010

June 14-19, 2010

Villasimius, Italy

${ }^{*}$ Speaker. 


\section{Introduction}

Creating hadrons usually forms the heart of many calculations carried out by lattice practitioners, whether their physics goals include spectroscopy [1], structure or matrix element determinations. In a Monte Carlo computation on a Euclidean lattice, the signal relevant to hadronic physics in a correlation function usually falls exponentially, and is rapidly dominated by statistical fluctuation. Operators that predominantly create low-lying energy eigenstates are thus very useful and improve the quality of measurements exponentially. The most useful tool in building a good creation operator is smearing: rather than defining a creation operator as a function on the bare fields in the lagrangian, they are first filtered to remove fluctuations at short distances that are less important in the low-energy state.

The task of computing the correlation function involving quarks is made difficult by the grassmann nature of the fermion fields in the lattice path integral. Direct manipulation of these fields is not computationally feasible. Instead, the fields are integrated analytically, leaving non-local functions of the gauge fields alone.

A method that provided a smearing scheme combined with a new approach to the computational problem of measuring quark propagation would be very valuable to anyone interested in making hadrons on the lattice and studying their properties. In this review, I describe a new framework called "distillation" [2]. that attempts to address both issues. It defines a smearing that does a good job of projecting onto the lowest energy eigenstates while facilitating easier evaluation of arbitrarily complicated correlation functions. In its simplest implementation, the technique suffers from poor volume scaling. Stochastic estimation is however seen to be very effective in reducing the computing budget dramatically and enabling large volume calculations. The use of a small vector space to capture the confinement-scale physics improves the convergence of these stochastic estimators.

\section{Quark smearing}

Consider computing the energy of an eigenstate of the Hamiltonian of a confining field theory such as QCD. In a Euclidean space-time metric, this requires a determination of the correlation function between a creation and annihilation operator $\Phi^{\dagger}$ and $\Phi$ that are localised in time;

$$
C\left(t^{\prime}, t\right)=\left\langle\Phi\left(t^{\prime}\right) \Phi^{\dagger}(t)\right\rangle .
$$

Inserting a complete set of eigenstates of the Hamiltonian with $\hat{H}|k\rangle=E_{k}|k\rangle$ gives the well-known expression for this correlation function as a sum of contributions from all modes;

$$
C\left(t^{\prime}, t\right)=\sum_{k=0}^{\infty}\left|\left\langle k\left|\Phi^{\dagger}\right| 0\right\rangle\right|^{2} e^{-E_{k}\left(t^{\prime}-t\right)}
$$

Here, $\left\langle k\left|\Phi^{\dagger}\right| 0\right\rangle$ is the matrix element detailing how effectively mode $|k\rangle$ is excited from the vacuum by creation operator $\Phi^{\dagger}$. To extract properties of low-lying states, it is crucial then to construct operators that overlap predominantly with these lightest modes (i.e. for which this element is large for low values of $k$ and suppressed for higher values). This yields a correlator which reaches its asymptotic form at earlier time separations, which in turn helps more statistically accurate determinations of energies. 
Our freedom in these calculations comes with the choice of $\Phi^{\dagger}$. Once we have determined how operators that transform irreducibly under the representations of the relevant symmetry groups must behave, we can choose to write any function of the fields on a time-slice in order to create the state of interest. Smearing is a very widely used method of helping in this procedure. Rather than applying the creation operator directly to the variables in the path-integral, a smoothing process is applied first. This process is chosen to filter out highly fluctuating modes, which are not relevant for creating hadrons, while maintaining as many symmetry properties of the fields as possible. One very widely used technique starts from the simplest representation of the gauge-covariant threedimensional laplacian.

$$
-\nabla_{x y}^{2}(t)=6 \delta_{x y}-\sum_{j=1}^{3}\left(\tilde{U}_{j}(x, t) \delta_{x+\hat{\jmath}, y}+\tilde{U}_{j}^{\dagger}(x-\hat{\jmath}, t) \delta_{x-\hat{\jmath}, y}\right)
$$

and where it is usually helpful to use gauge fields $\tilde{U}$ that have themselves been smeared using a covariant link-smearing scheme [3,4]. The Gaussian smeared quark field $\tilde{\psi}$ is then defined as

$$
\tilde{\psi}_{x}(t)=J_{x y}(t) \psi_{y}(t)
$$

with the linear operator $J$ given by

$$
J_{\sigma, n_{\sigma}}(t)=\left(1+\frac{\sigma \nabla^{2}(t)}{n_{\sigma}}\right)^{n_{\sigma}} .
$$

Note that smearing need not be a linear operation (it is not for the gauge fields), but this is a very useful property for quark fields as it keeps subsequent evaluation of correlation functions via Wick contractions simple. We will keep to this restriction here. When $n_{\sigma}$ is large, Euler's limit gives

$$
\lim _{n_{\sigma} \rightarrow \infty} J_{\sigma, n_{\sigma}}(t)=\exp \left(\sigma \nabla^{2}(t)\right)
$$

and we see that rapidly fluctuating modes are exponentially suppressed by this operator. Figure 1 shows the spectrum of this operator on a typical small production lattice. One observation is clear; most modes are extremely highly suppressed, and the operators is effectively very low rank, with effective support on $\mathscr{O}(100)$ modes out of $16^{3} \times 3=12,288$.

\subsection{Distillation}

Recognising that effective smearing operators are very low rank suggests making an explicit replacement. Remember we are free to choose any smearing operator as long as it does not break symmetries and remains well localised in time. Low-rank operators are often easier to manipulate numerically and this will prove to be the case with quark propagation.

With access to all $N_{\mathrm{ev}}=N_{c} \times N_{s}$ eigenvectors, $v$ and corresponding eigenvalues, $\lambda \geq 0$ of the gauge-covariant lattice laplacian on a particular time-slice, a spectral representation of the gaussian smearing operator $J$ can be written;

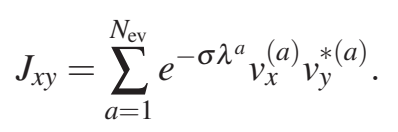




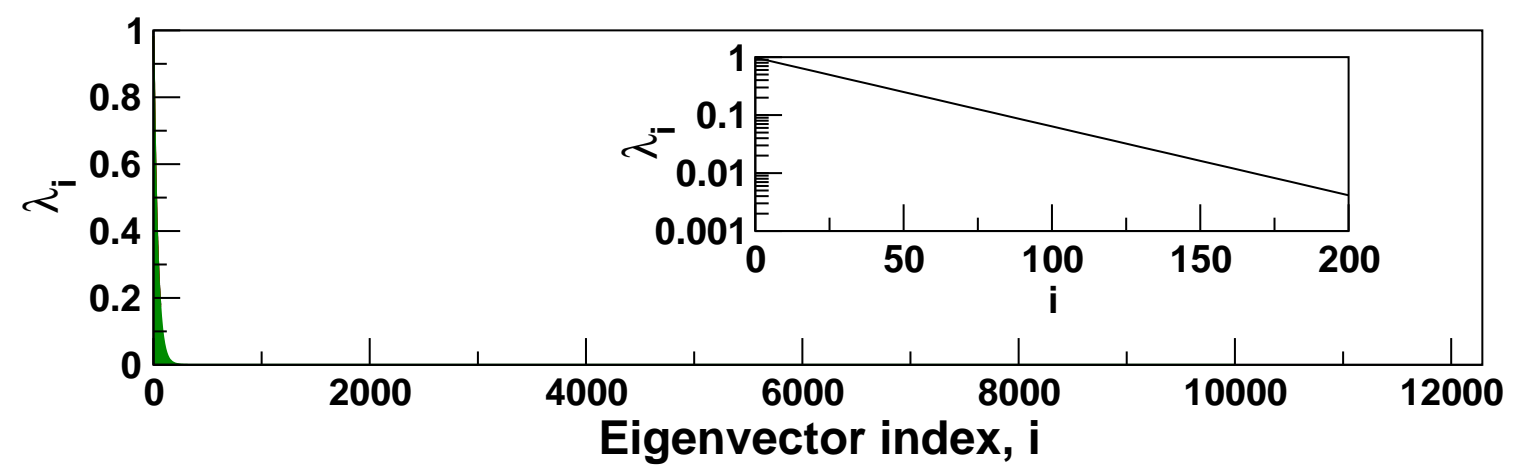

Figure 1: The spectrum of the Gaussian smearing operator on a $16^{3}$ spatial volume. The main panel shows the raw data barely visible in green, while the inset reproduces the first 200 modes on a logarithmic scale. All but the first $\mathscr{O}(100)$ modes are very highly suppressed.

Note that the numerical problem of finding at least a subset of the lowest of these modes is reasonably tractable; the three-dimensional laplace operator is hermitian and non-negative, so well suited to the Lanczos algorithm.

Now redefine this smearing by truncating at a low mode, $N_{\mathscr{D}} \ll N_{\mathrm{ev}}$. Applying this explicitly constructed low-rank operator will be called "distillation" [2]. Figure 1 suggests the number of modes needed to capture confinement physics on a $2 \mathrm{fm}$ lattice is less than 100 . In principle, there would be an ambiguity defining this operator if two or more modes straddling $N_{\mathscr{D}}$ were degenerate, since it would be unclear which to keep in the truncated sum. In practise, this ambiguity does not arise suggesting these degenerate time-slices form a set with zero probability measure in the partition function. As another simplification, the exponential weights in the truncation can be dropped and empirically this is seen to have very little effect on hadronic correlation functions once $N_{\mathscr{D}}$ is fixed at a reasonably small value. The distillation operator is then simply written

$$
\square(t)=V(t) V^{\dagger}(t)
$$

with $V$ an $\left(N_{c} \times N_{s}\right) \otimes N_{\mathscr{D}}$ matrix. The distilled operator inherits all the symmetries of the lattice laplacian. It is rotationally and translationally invariant (provided there are none of the degeneracy problems described above). It is also covariant under gauge transformations. These symmetries make it an appropriate choice for a quark smearing operator. Figure 2 shows the modulus of a distilled source measured at points separated from the source both on- and off-axis on the lattice. Continuum rotational invariance is seen. The different curves correspond to different choices of the cut-off on modes in the sum.

The benefit of this modification to the smearing operator becomes apparent only when numerical measurements of hadronic observables on the lattice are considered. To take an example, after integrating out the quark fields, the correlation function of an isovector meson created by a general operator on distilled fields $\bar{u} \square \Gamma^{\dagger} \square d$ can be written

$$
C_{M}\left(t^{\prime}-t\right)=\left\langle\operatorname{Tr}\left(\square \Gamma\left(t^{\prime}\right) \square M^{-1}\left(t^{\prime}, t\right) \square \Gamma^{\dagger}(t) \square M^{-1}\left(t, t^{\prime}\right)\right)\right\rangle .
$$

Here, the notation for operator $\Gamma(t)$ is meant to describe an arbitrarily complicated function of the gauge fields on time-slice $t$. Spin, colour and spatial indices are suppressed. At first sight, this 


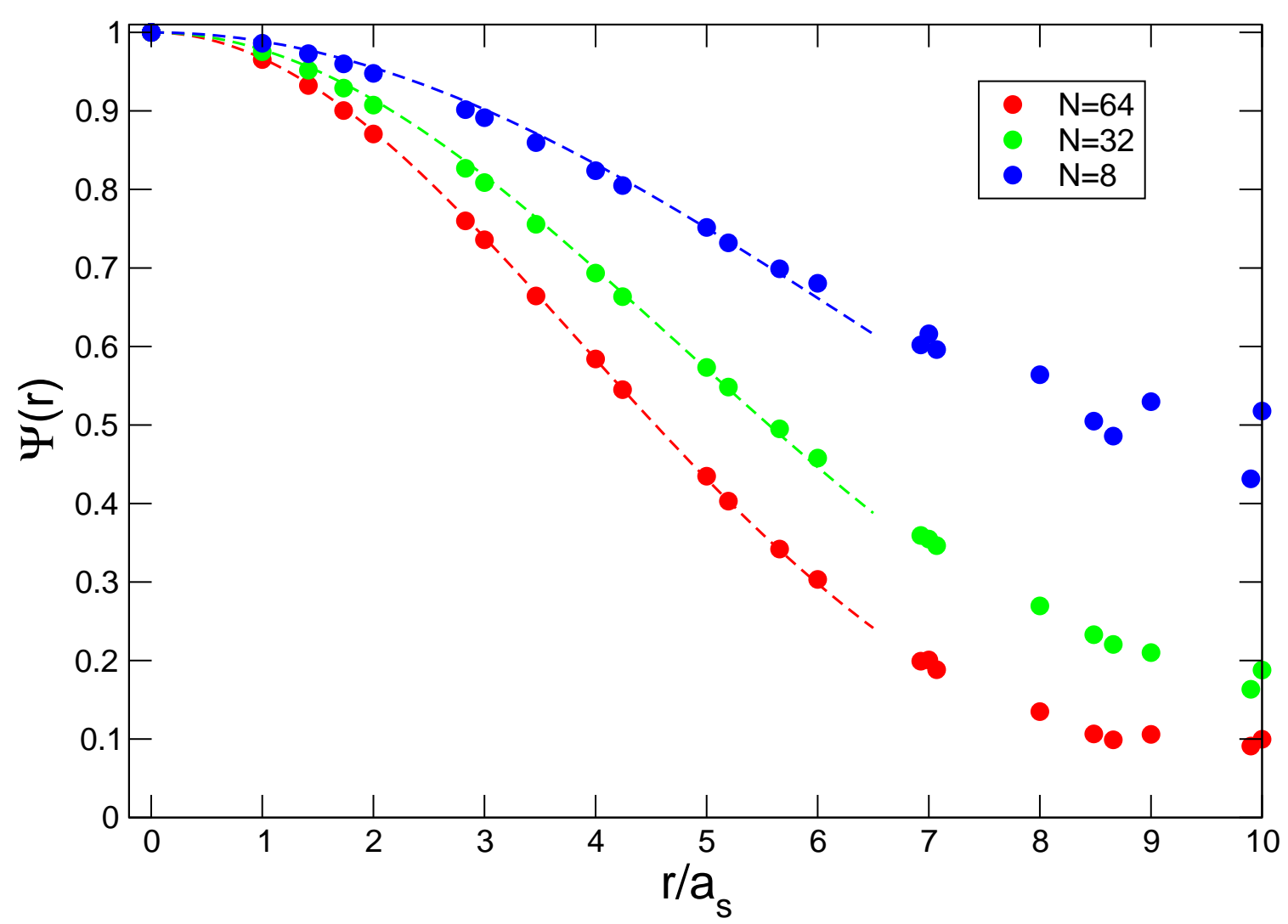

Figure 2: The spatial distribution of a distilled source for three different sizes of distillation spaces (taken from [2]). The scatter between points at large separation can be attributed to the finite extent of the lattice.

trace appears computationally intractible, since it involves summation over all spatial points on the time-slices $t$ and $t^{\prime}$ and so would require information on the quark propagator from all points on $t$ to all points on $t^{\prime}$. The usual means of evaluating an expression of this form is to restrict the choice of operator at the source to excite quark fields at a single point only and compute just the restricted set of columns of the quark propagator needed to measure this correlator.

On closer inspection though, the distilled expression is a trace over a much smaller space (of dimension $N_{\mathscr{D}} \times N_{\gamma}$ and the correlation function becomes

$$
C_{M}\left(t^{\prime}-t\right)=\left\langle\operatorname{Tr}\left(\Phi\left(t^{\prime}\right) \tau\left(t^{\prime}, t\right) \Phi^{\dagger}(t) \tau\left(t, t^{\prime}\right)\right)\right\rangle
$$

with

$$
\Phi(t)=V^{\dagger}(t) \Gamma(t) V(t)
$$

and

$$
\tau\left(t, t^{\prime}\right)=V^{\dagger}(t) M^{-1}\left(t, t^{\prime}\right) V\left(t^{\prime}\right) .
$$

$\Phi$ and $\tau$ are relatively small matrices here. Two observations are useful; first there has been no need to restrict the source to a particular point and translational invariance is maintained, enabling operators of definite momentum to be used at both source and sink. Second, the observation that the creation operator is encapsulated in $\Phi$ alone means correlations between arbitrary sources can be 
computed with no need to recompute quark propagators (encoded solely in $\tau$ ). This is very useful for variational calculations for example, where a large basis of different source/sink operators is used and where correlation functions involving all possible combinations of sources and sinks must be computed.

More complicated diagrams are made tractable too. The isoscalar mesons can be investigated by evaluating disconnected terms that arise in the Wick contraction and these are facilitated by the method. If hadron scattering and decays are to be studied, diagrams that involve creation of multiple mesons (each with well-defined momentum) are needed. Since quark propagation from all points on the time-slice is computed, these measurements are made possible too. Examples of practical calculations involving just such diagrams will be presented later. Baryons can naturally be computed following a similar recipe.

\section{First numerical results}

Distillation has been used in a number of large-scale calculations carried out by the Hadron Spectrum Collaboration. The spectrum of isovector mesons was computed [5, 6] and the excitations of the nucleon, $\Delta$ and $\Omega$ baryons were investigated in Ref. [7]. The spectrum of isovector mesons with $m_{\pi}=500 \mathrm{MeV}$ is reproduced in Fig 3. In this determination, large variational bases

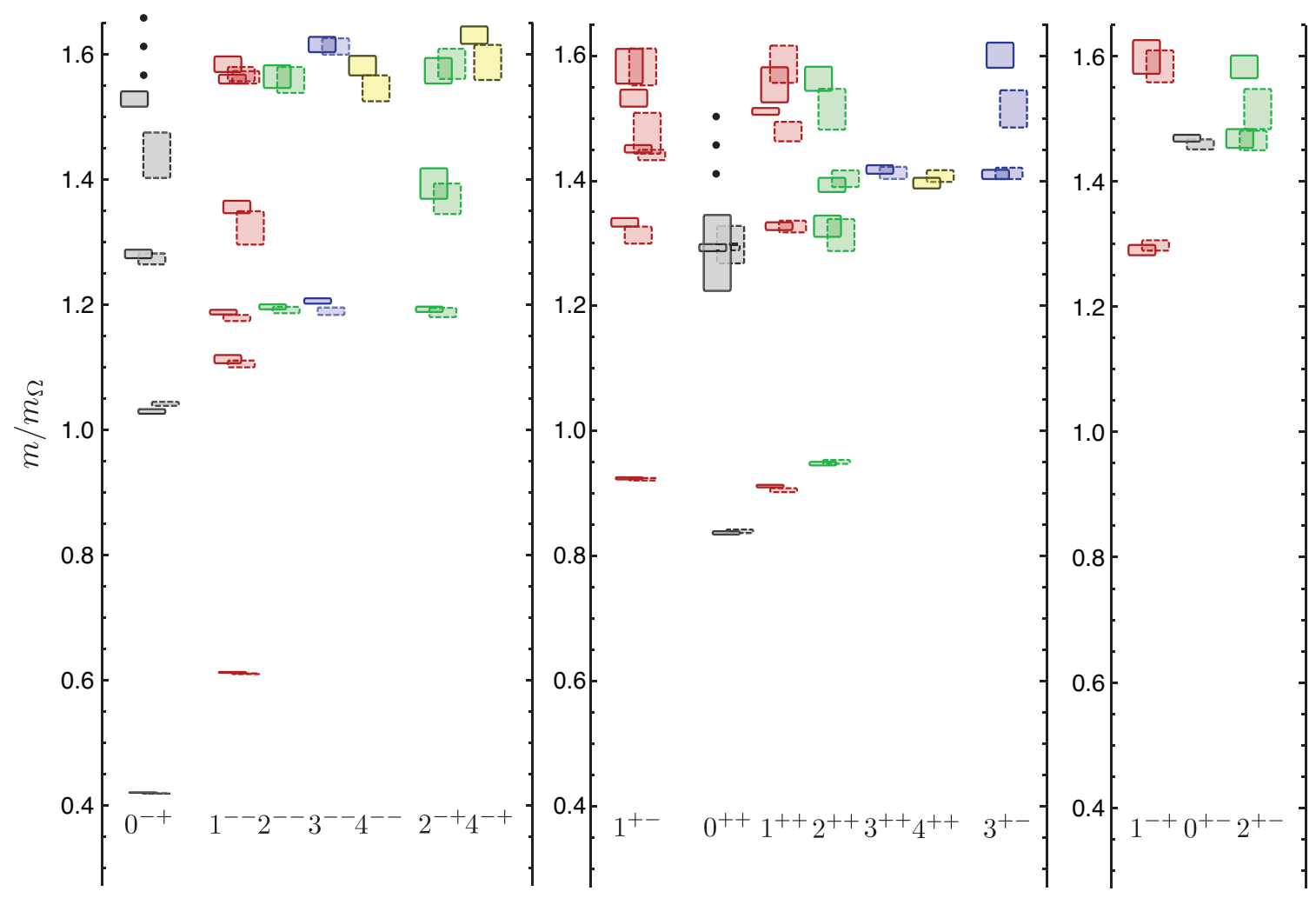

Figure 3: The spectrum of light isovector mesons with $m_{\pi} \approx 700 \mathrm{MeV}$ determined using distilled quark fields. Spin identification was carried out using derivative based operator construction.

were used to investigate many spin channels including the spin-exotic hybrid mesons. The spectrum of excited nucleons is presented in Figure 4. This investigation also made use of a carefully 

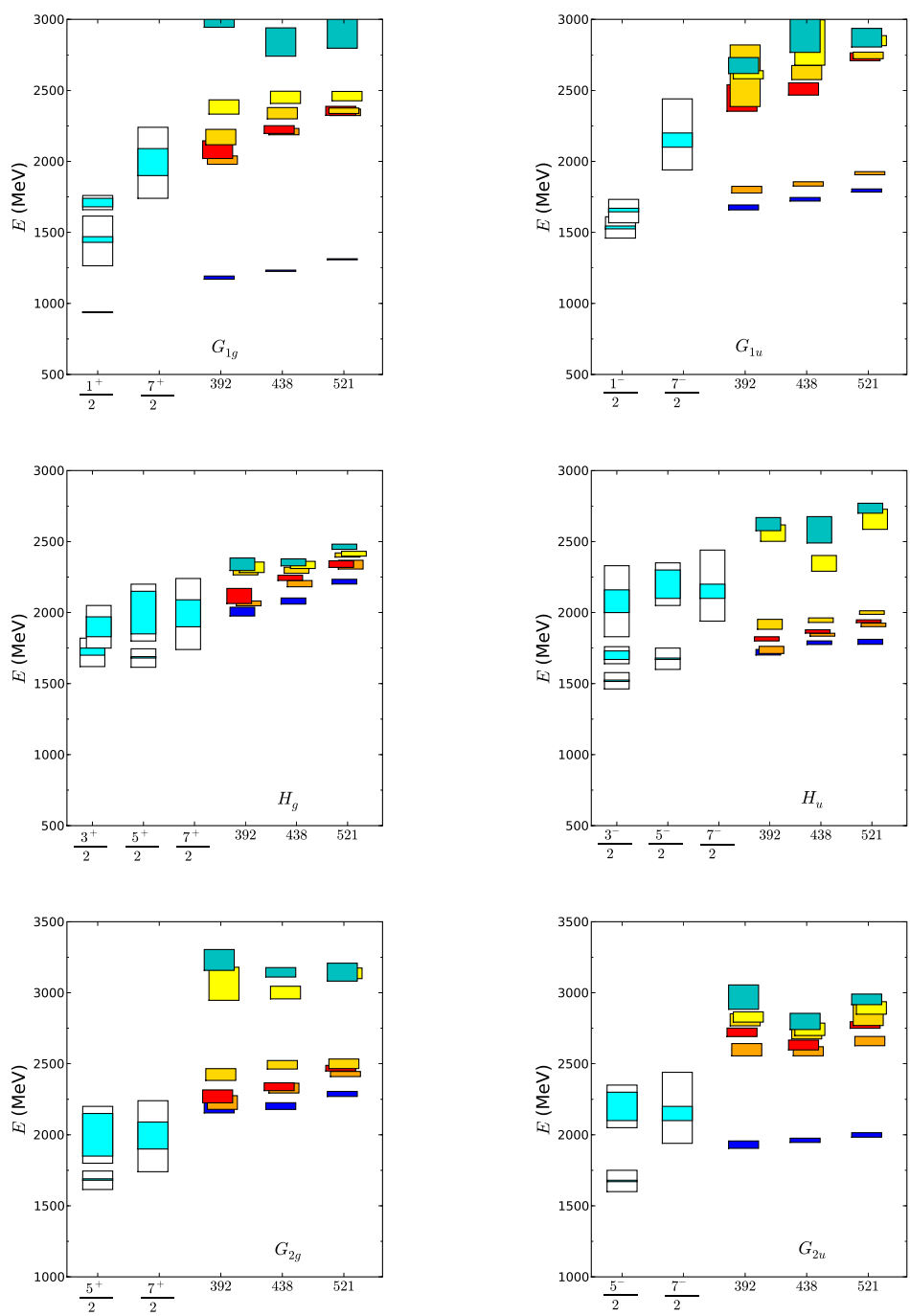

Figure 4: The spectrum of nucleons determined using distillation [7].

constructed variational basis to determine the excitation spectrum. The benefit in precision arising from distillation is clear from Fig 5, where data from a point-propagator calculation is compared. Subsequently investigations of $I=2$ scattering [8] and the isoscalar meson spectrum [9] have been carried out Distillation gives excellent resolution of disconnected contributions to the correlation function as can be seen in Figure 6, which shows data from about 500 gauge field configurations. These computations were however very expensive, requiring millions of solutions to the linear system for quark propagation to be evaluated. This was only made feasible by use of GPGPUs and the QUDA library [10]. The end result of this determination is shown in Figure 7. An unprecedented view of the spectrum of isoscalar mesons was obtained, giving detailed data on states up to spin 4 , and including isoscalar spin-exotic hybrids. The mass of the $\eta^{\prime}$ resonance is resolved in this calculation at about the percent-level statistical accuracy. The $\rho-\omega$ mass splitting is resolved to MeV-level precision. 


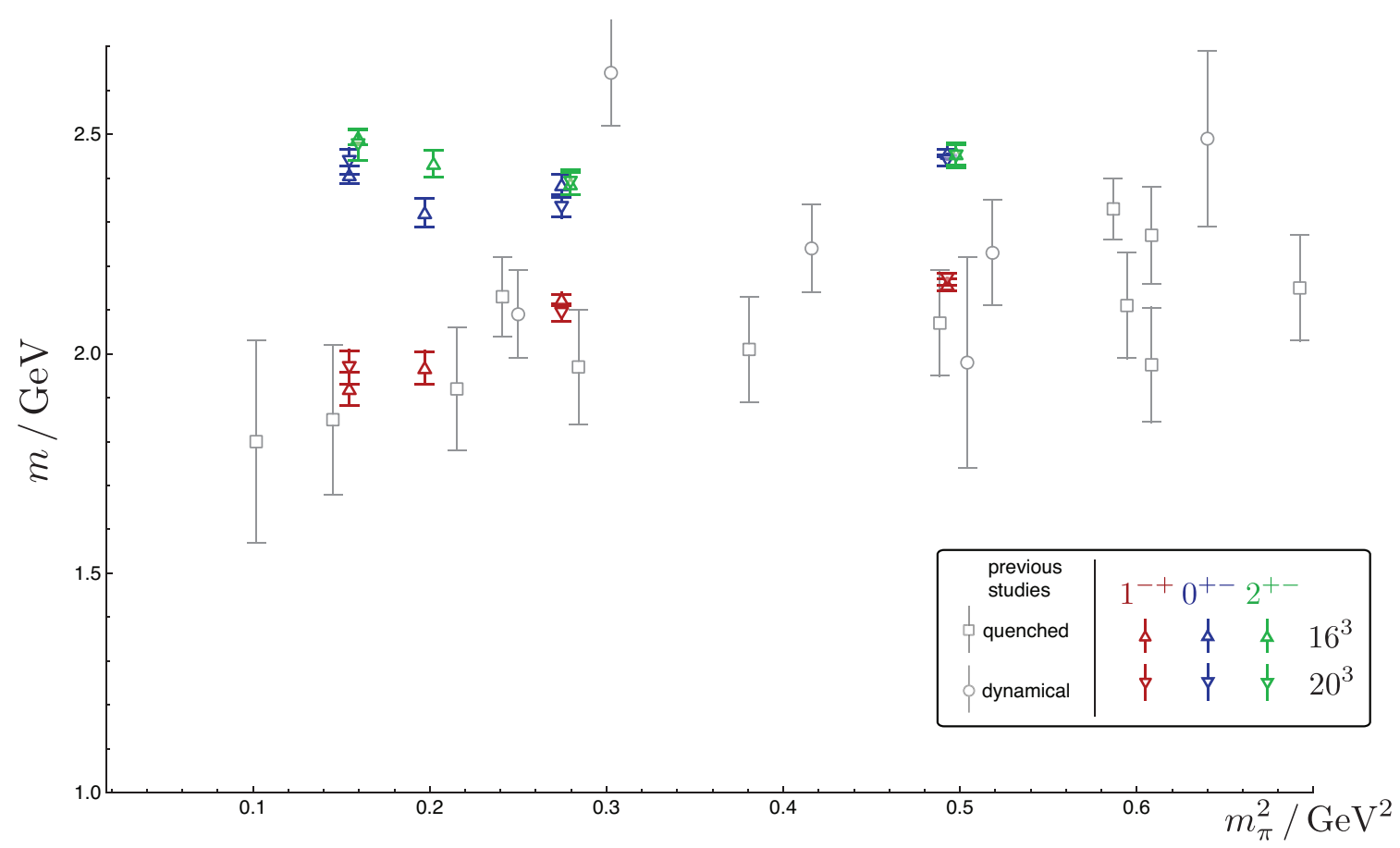

Figure 5: Comparison of statistical precision of spin-exotic meson energies, determined using point-to-all methods and distillation. Point-to-all data are taken from Refs [11, 12, 13, 14, 15, 16]

\section{The scaling problem and stochastic estimation}

There is a substantial drawback with direct numerical implementation of the method. The number of modes $N_{\mathscr{D}}$ needed to keep a constant resolution at the hadronic scale grows with the spatial volume. A simple means of understanding this is to imagine the free theory, where the eigenvectors of the laplacian are the Fourier modes. If structure up to some fixed momentum scale $p^{*}$ is required then as $L$, the extent of the lattice grows the number of modes below $p^{*}$ grows in inverse proportion to $V=L^{3}$ as the density of states increases.

Note that this issue is entirely independent of the lattice spacing $a$ and is governed by lowenergy dynamics. As the continuum limit is approached, it is to be expected that the number of modes needed will not vary significantly although this has yet to be tested numerically. It is only the large- $V$ limit that presents a problem for the computer budget. Nevertheless this is a significant problem that makes large simulations extremely expensive. It implies that the computing cost for evaluating the quark propagator from all $N_{\mathscr{D}}$ sources must grow at least like $V^{2}$. To make the issue worse, consider how expensive contracting quark propagators to form meson and baryon correlation functions is. As we have seen, evaluating a meson correlator is equivalent to computing the trace of a product of $N_{\mathscr{D}} \times N_{\mathscr{D}}$ matrices and the cost of this grows like $N_{\mathscr{D}}^{3} \equiv V^{3}$. For baryons, the problem is worse still, with a $\mathscr{O}\left(V^{4}\right)$ dependency.

The computing cost can be dramatically reduced and brought under control by using a stochastic estimate of the correlation function. The important advantage that distillation has enabled is that these estimate can be constructed within the space of distilled fields, rather than the much larger space of unsmeared lattice fields. This leads to substantial benefits in variance reduction. These 


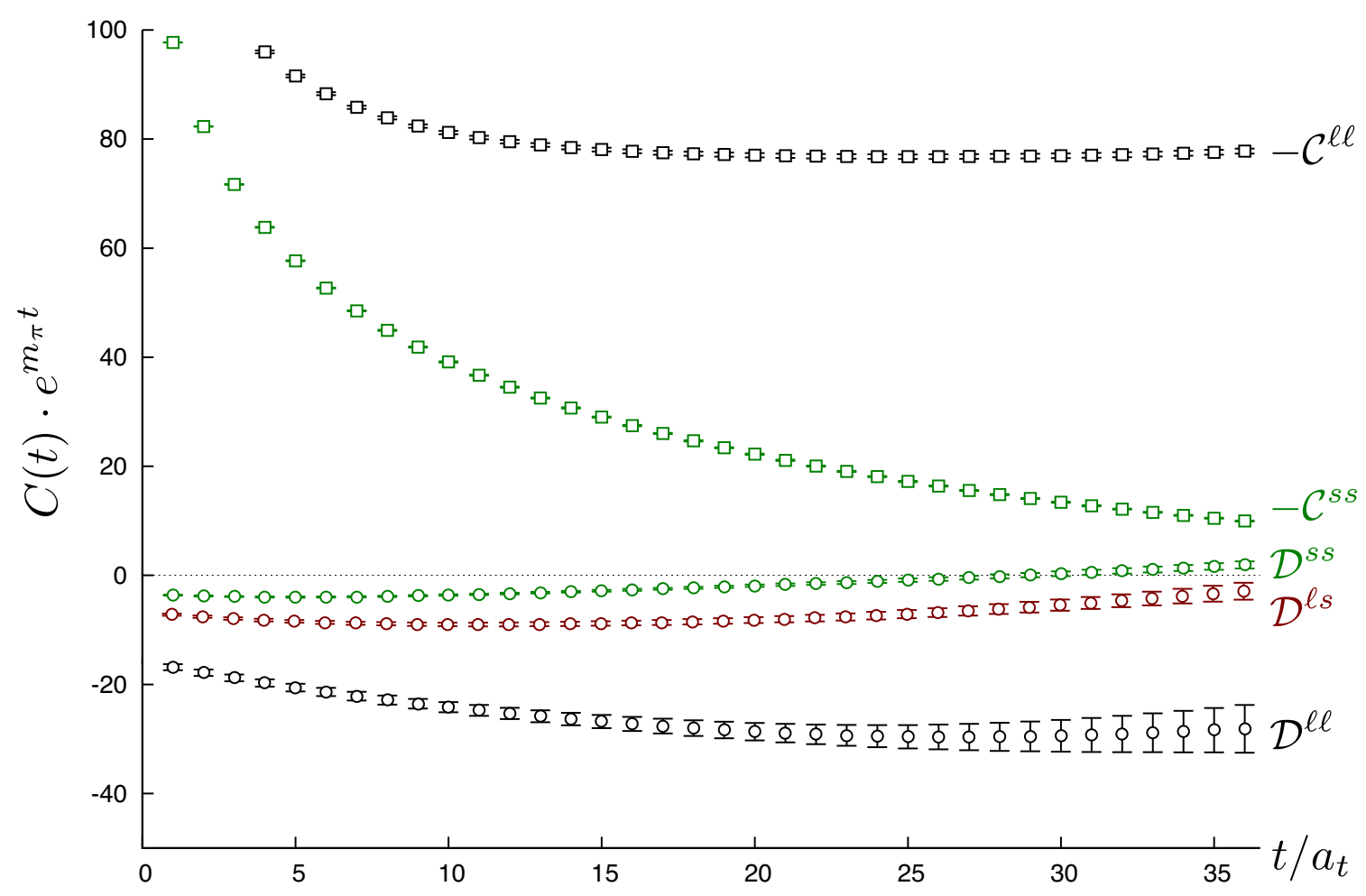

Figure 6: The diagrams contributing to the correlation function of the light, psuedoscalar isoscalar mesons, $\eta$ and $\eta^{\prime} . \mathscr{C}$ and $\mathscr{D}$ indicate connected and disconnected contributions and the hidden flavour in the source and sink is indicated by the superscripts $l$ and $s$ respectively.

computations start from the usual place; a stochastic representation of the identity matrix is written as the outer product of two vectors filled with independent random numbers: $I=E\left[\eta \eta^{\dagger}\right]$. In this case, these vectors lie in the space of distilled fields on a time-slice, so they are relatively small. Now the distillation operator can be written

$$
\square=E\left[V \eta \eta^{\dagger} V^{\dagger}\right]
$$

The ideas of "dilution" $[17,18]$ are very useful again to control the variance of these estimators. In tests performed to date, dilution is carried out by collecting the vectors into sub-sets and adding noise into these smaller spaces in turn. Now the distillation operator is written

$$
\square=\sum_{q} E\left[V P^{(q)} \eta \eta^{\dagger} P^{(q)} V^{\dagger}\right]
$$

with $P^{(q)}$ a projection operator into partition $q$ of the distillation space so that $P^{(q) 2}=P^{(q)}$ and $\sum_{q} P^{(q)}=I$. For details and a practical implementation, see Ref [19]. Using the distillation space as the place to inject noise and cut costs has a substantial benefit as is seen in Fig 8 .

At this meeting, first results that exploit this stochastic estimation approach to evaluating distilled correlation functions were presented [20, 21, 22]. More information can be seen in other conference presentations from 2010: [23, 24, 25, 26]. Recently, a more comprehensive investigation was completed and is presented in [19]. 


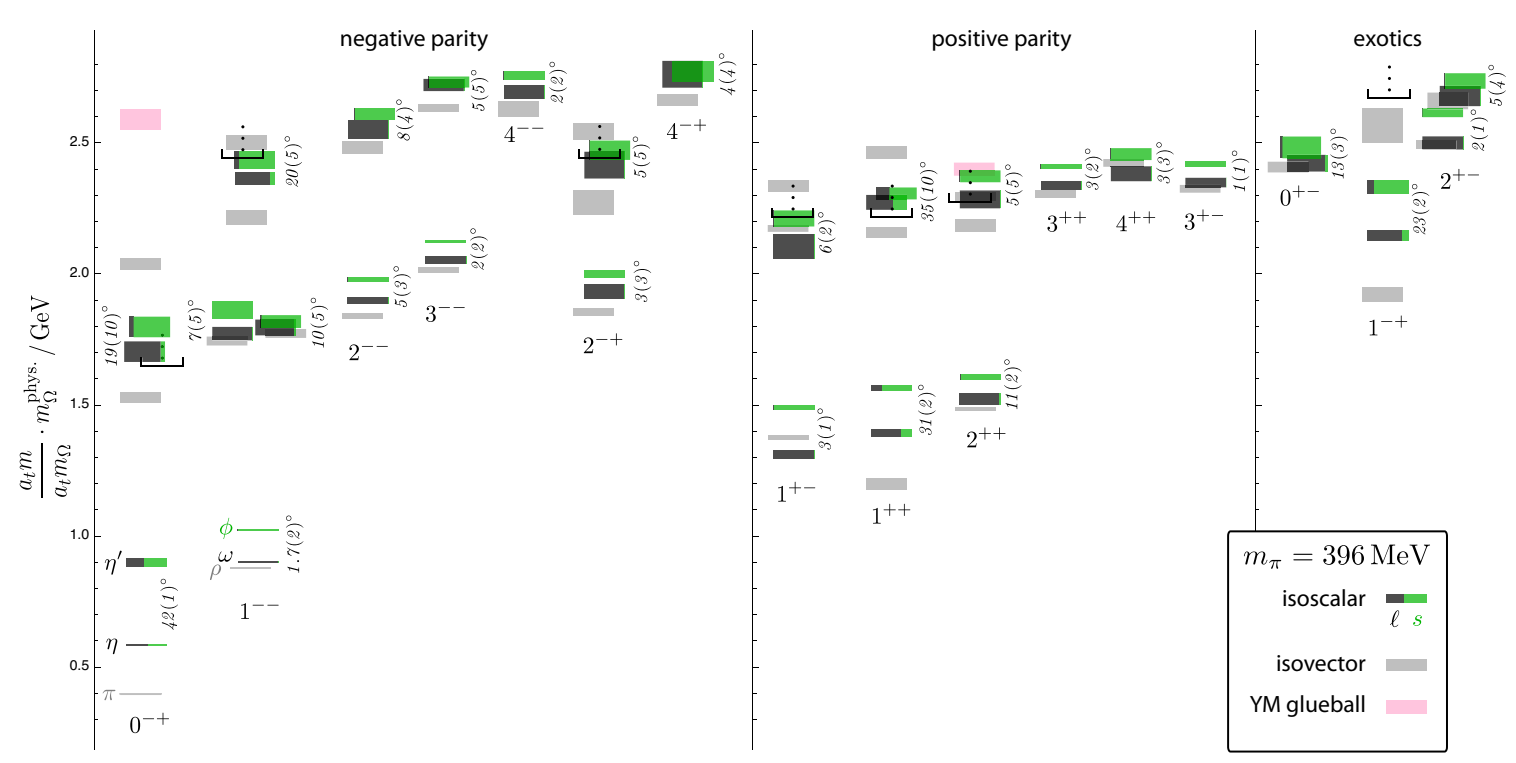

Figure 7: The spectrum of isoscalar mesons when $m_{\pi}=396 \mathrm{MeV}$. Flavour mixing angles are indicated via the relative amounts of black and green on the bars. The corresponding isovector meson spectrum is superimposed in grey and the Yang-Mills glueball states are indicated in pink.

\section{Summary}

A simple observation about the quark-field smearing process suggested a new approach to help tackle previously difficult problems in lattice hadronic physics. In smearing, a very substantial fraction of the modes of the field are almost entirely eliminated. Since smearing operators can be defined arbitrarily (within a set of constraints), a helpful choice is to use an explicitly low-rank operator with support in a small vector space of smooth fields on each time-slice of the lattice. This is distillation.

On small lattices (below about $2 \mathrm{fm}$ box-length), the number of modes needed to create mesons and hadrons is sufficiently small that all elements of the restricted propagator can be computed at a manageable cost (in comparison to generating dynamical gauge field configurations). Bigger volumes need too many inversions for this to be tractable but first data from stochastic estimation experiments strongly suggest this problem can be ameliorated.

With all elements of the restricted propagator (or at least a good stochastic estimator of them) to hand, a wide range of operators become available. A large basis of creation operators can be inserted and all elements of the correlation matrix measured. This enables a variational calculation to be performed, giving information on excited states and states with higher spin. Hybrid mesons are measured with unprecedented accuracy. The correlation functions for isoscalar states, which have contributions from disconnected diagrams can be computed to good precision. Multi-hadron states can be computed, and this conference saw a number of presentations demonstrating this application.

While it has already proven useful in production measurements, more work to fine-tune the method might be beneficial. Using eigenmodes of the three-dimensional lattice laplace operator on 


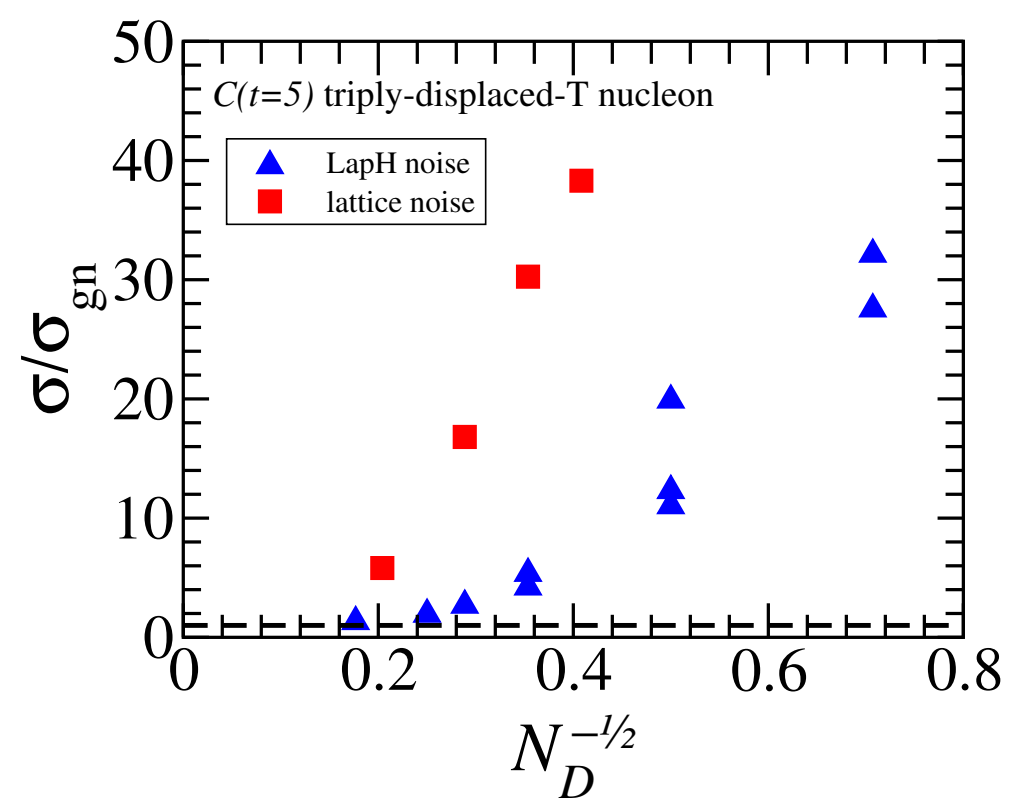

Figure 8: A comparison between variance in estimators for the correlation function for a nucleon computed using noise inserted into the space of unsmeared quark fields (red data) and distilled quark fields (blue). A clear benefit to using stochastic estimators in the distillation space is seen. [19].
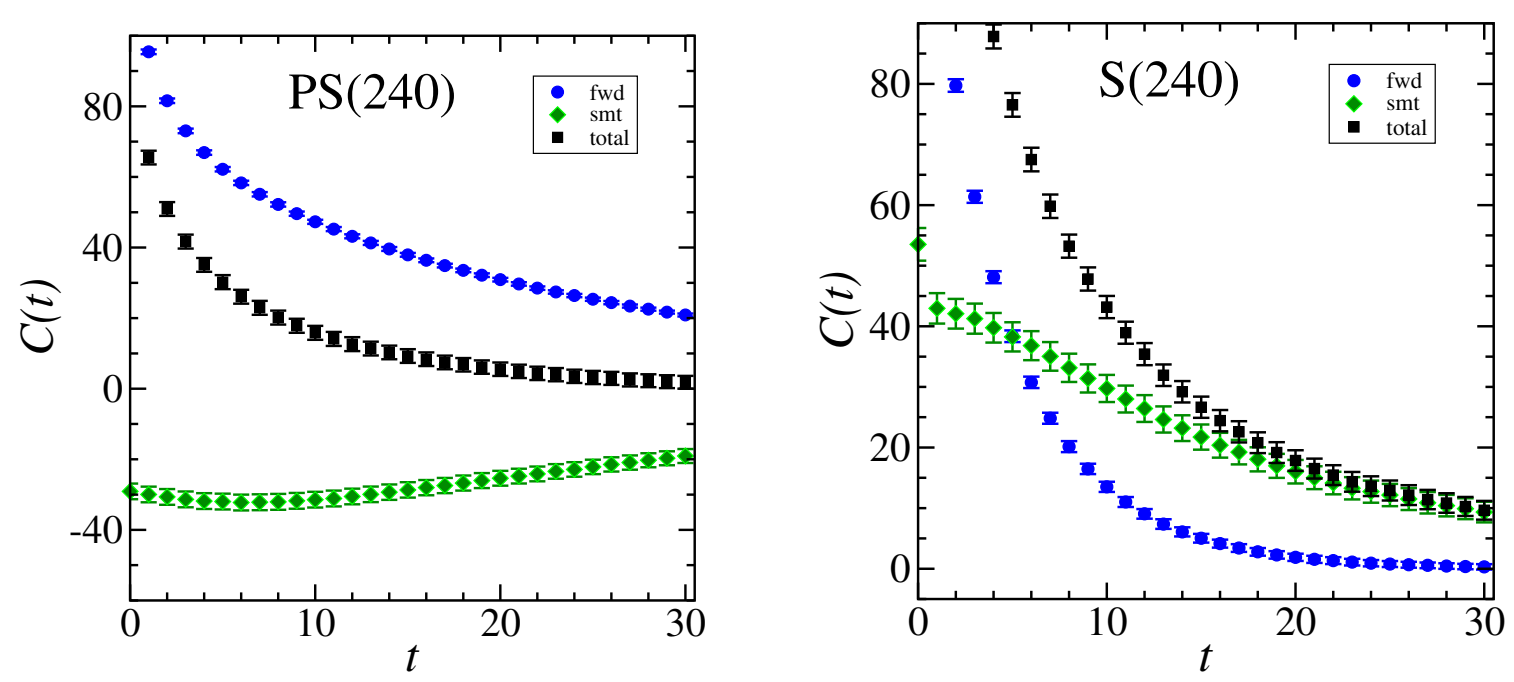

Figure 9: Correlation functions of pseudoscalar and scalar isoscalar mesons constructed from distilled sources and estimated stochastically for $m_{\pi}=240 \mathrm{MeV}$ [19].

each time-slice has been shown to be a very good starting point in defining smooth, gauge covariant modes but it is by no means unique and there is no deep reason to expect it is optimal. Other choices should be considered.

The big problem with direct computations is the scaling with the lattice volume. While some improvements in signal quality come from working in a bigger volume, the naive expectation is that the number of modes needed grows like the three-dimensional volume of a time-slice. The basis needs to resolve features at some fixed confinement scale, and the number of these features that can 
fit into a box grows in direct proportion. Numerical experiments bear out this simple reasoning. The use of stochastic estimators, with the noise put directly into the distillation space appears to solve this problem. A number of results from first tests were presented at this meeting and illustrate this conclusion.

The framework promises to be very useful when states that resemble multiple hadrons are to be constructed. These operators appear to be crucial in determinations of scattering properties and resonances on the lattice. The implementation and exploitation of distillation in these calculations is under investigation.

\section{Acknowledgements}

I would like to thank all my collaborators in the Hadron Spectrum Collaboration. I am very grateful to the theory division at Jefferson Laboratory for their generous hospitality during the start of this project. This work is supported by Science Foundation Ireland under grant number 07/RFP/PHYF168 and by the Research Executive Agency (REA) of the European Union under grant agreement number PITN-GA-2009-238353 (ITN STRONGnet).

\section{References}

[1] C. Hoelbling, PoS LATTICE2010 (2010) 011 [arXiv:1102.0410 [hep-lat]].

[2] M. Peardon et al. [Hadron Spectrum Collaboration], Phys. Rev. D 80 (2009) 054506 [arXiv:0905.2160 [hep-lat]].

[3] M. Albanese et al. [APE Collaboration], Phys. Lett. B 192 (1987) 163.

[4] C. Morningstar and M. J. Peardon, Phys. Rev. D 69 (2004) 054501 [arXiv:hep-lat/0311018].

[5] J. J. Dudek, R. G. Edwards, M. J. Peardon, D. G. Richards and C. E. Thomas, Phys. Rev. Lett. 103 (2009) 262001 [arXiv:0909.0200 [hep-ph]].

[6] J. J. Dudek, R. G. Edwards, M. J. Peardon, D. G. Richards and C. E. Thomas, Phys. Rev. D 82 (2010) 034508 [arXiv:1004.4930 [hep-ph]].

[7] J. Bulava et al., Phys. Rev. D 82 (2010) 014507 [arXiv:1004.5072 [hep-lat]].

[8] J. J. Dudek, R. G. Edwards, M. J. Peardon, D. G. Richards and C. E. Thomas, Phys. Rev. D 83 (2011) 071504 [arXiv:1011.6352 [hep-ph]].

[9] J. J. Dudek, R. G. Edwards, B. Joo, M. J. Peardon, D. G. Richards and C. E. Thomas, arXiv:1102.4299 [hep-lat].

[10] R. Babich, M. A. Clark and B. Joo, arXiv:1011.0024 [hep-lat].

[11] C. Bernard et al., Phys. Rev. D 68 (2003) 074505 [arXiv:hep-lat/0301024].

[12] C. W. Bernard et al. [MILC Collaboration], Phys. Rev. D 56 (1997) 7039 [arXiv:hep-lat/9707008].

[13] P. Lacock and K. Schilling [TXL collaboration], Nucl. Phys. Proc. Suppl. 73 (1999) 261 [arXiv:hep-lat/9809022].

[14] P. Lacock, C. Michael, P. Boyle and P. Rowland [UKQCD Collaboration], Phys. Lett. B 401 (1997) 308 [arXiv:hep-lat/9611011]. 
[15] C. McNeile and C. Michael [UKQCD Collaboration], Phys. Rev. D 73 (2006) 074506 [arXiv:hep-lat/0603007].

[16] J. N. Hedditch, W. Kamleh, B. G. Lasscock, D. B. Leinweber, A. G. Williams and J. M. Zanotti, Phys. Rev. D 72 (2005) 114507 [arXiv:hep-lat/0509106].

[17] S. Bernardson, P. McCarty and C. Thron, Comput. Phys. Commun. 78 (1993) 256.

[18] J. Foley, K. Jimmy Juge, A. O’Cais, M. Peardon, S. M. Ryan and J. I. Skullerud, Comput. Phys. Commun. 172 (2005) 145 [arXiv:hep-lat/0505023].

[19] C. Morningstar, J. Bulava, J. Foley, K. J. Juge, D. Lenkner, M. Peardon and C. H. Wong, arXiv:1104.3870 [hep-lat].

[20] J. Bulava, J. Foley, K. J. Juge, C. J. Morningstar, M. J. Peardon and C. H. Wong, PoS LATTICE2010 (2010) 110 [arXiv:1011.5277 [hep-lat]].

[21] J. Foley, C. H. Wong, J. Bulava, K. J. Juge, D. Lenkner, C. Morningstar and M. Peardon, arXiv:1011.0481 [hep-lat].

[22] J. Bulava et al., PoS LATTICE2010 (2010) 129 [arXiv:1011.1509 [hep-lat]].

[23] J. Bulava, K. J. Juge, C. J. Morningstar, M. J. Peardon and C. H. Wong, arXiv:0911.2044 [hep-lat].

[24] S. Cohen et al., arXiv:0911.3373 [hep-lat].

[25] C. Morningstar et al., "The excited hadron spectrum in lattice QCD using a new method of estimating quark propagation,” AIP Conf. Proc. 1257 (2010) 779 [arXiv:1002.0818 [hep-lat]].

[26] J. Foley, J. Bulava, K. J. Juge, C. Morningstar, M. Peardon and C. H. Wong, AIP Conf. Proc. 1257 (2010) 789 [arXiv:1003.2154 [hep-lat]].

[27] R. G. Edwards, J. J. Dudek, D. G. Richards and S. J. Wallace, arXiv:1104.5152 [hep-ph]. 